\title{
Effect of Diagnosis on Variability of ICU Patients in Insulin Sensitivity *
}

\author{
Tamás Ferenci*, Levente Kovács*, Balázs Benyó*, \\ Aaron Le Compte ${ }^{* *}$ Geoffrey M. Shaw ${ }^{* * *}$ \\ Geoffrey J. Chase** \\ * Department of Control Engineering and Information Technology, \\ Budapest University of Technology and Economics, Budapest, Hungary \\ (e-mails: \{ferenci, lkovacs, bbenyo\}@iit.bme.hu) \\ ** University of Canterbury, Dept of Mechanical Engineering, Centre \\ for Bio-Engineering, Christchurch, New Zealand \\ (e-mails: \{aaron.lecompte, geoff.chase\}@canterbury.ac.nz) \\ *** Depertment of Intensive Care, Christchurch Hospital, Christchurch, \\ New Zealand \\ (e:mail: geoff.shaw@cdhb.govt.nz)
}

\begin{abstract}
Tight glycemic control (TGC) in intensive care unit (ICU) patients represents an active research field as it has been proved its mortality and cost reduction effects. Previous works demonstrated that insulin sensitivity plays an important role in this question. The paper investigates by two defined metrics patient's variability in insulin sensitivity based on a previously introduced (ICING) glucose-insulin model. These metrics grasp the deviations of the actual insulin sensitivity data from their predictions; hence, characterizing a patient's variability at a given time. We also introduce and examine a way to characterize variability across longer time periods and across different patients. Investigations are applied to an actual longitudinal database consisting of $n=261$ patients with 47,836 hours of measurement in total, with patients grouped according to diagnosis groups formed from their Apache III codes. Data was also segregated according to time spent in ICU (in days). Kruskal-Wallis-test was then employed (separately for different days) to assess whether patients in different diagnosis groups exhibit significantly different variability. Differences were further analyzed with Tukey HSD post-hoc testing. Results show that insulin sensitivity decreases with time and that differences between diagnosis groups diminish. However, there are significant differences on the first two days of stay in the ICU according to one of the metrics, with cardiac patients being more variable and gastric patients (especially non-operative ones) being less variable.
\end{abstract}

Keywords: Insulin sensitivity, Patient variability, Statistical Analysis, Statistical Inference, Tight glycemic control.

\section{INTRODUCTION}

Stress induced hyperglycemia is a significant issue in critical care, affecting up to $30-50 \%$ of patients and increasing morbidity and mortality (Krinsley (2003) and McCowen et al (2001)). Controlling glycemia has proved difficult due to the associated risk of hypoglycemia when highly dynamic patients are treated with exogenous insulin (Griesdale et al (2009)). Both extremes, as well as glycemic variability, have been independently linked to increased morbidity and mortality (Bagshaw et al (2009), Egi et al (2006) and Krinsley (2008)), creating a difficult clinical

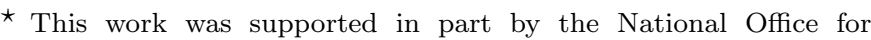
Research and Technology (NKTH), Hungarian National Scientific Research Foundation grant OTKA K82066. It is connected to the scientific program of the "Development of quality-oriented and harmonized $\mathrm{R}+\mathrm{D}+\mathrm{I}$ strategy and functional model at BME" project, supported by the New Hungary Development Plan (Project ID: TMOP-4.2.1/B-09/1/KMR-2010-0002).
}

problem to safely and effectively regulate glycemia to a physiologically and clinically safe range.

Glycemic control can reduce negative outcomes (Krinsley (2004), Chase et al (2008) and Berghe et al (2001)), but has proven difficult (Casaer et al (2011), Brunkhorst et al (2008) and Finfer et al (2009)). Only Chase et al (2008) reduced both mortality and hypoglycemia. However, inter- and intra- patient metabolic variability (Chase et al (2011)) makes good control difficult. Hence, glycemic targets have been raised (Moghissi et al (2009)).

Change in patient-specific insulin sensitivity drive outcome glycemic variability and hypoglycemic risk (Chase et al (2011)). However, cohort-based stochastic models (Lin et al (2008)) can be too conservative. It is the risk of sudden rises in insulin sensitivity that can result in a hypoglycemic event for a given insulin dose over a typical 3-4 hour measurement interval are of greatest concern. It is critical to determine the size and likelihood of these variations. 
This research statistically analyses insulin sensitivity variability as a function of diagnostic category and day of stay.

\section{MATERIAL AND METHODS}

To assess the effect of diagnosis and length-of-stay on the variability of a clinically validated model-based insulin sensitivity metric $(S I)$ ?the ending of the sentence is missing here? The Markov-approximation provides a model to predict future insulin sensitivity values: first, the present insulin sensitivity $(S I(n))$ is identified, then, the cohort model is used to predict the distribution of insulin sensitivity at the next time-point $\left(\hat{f}_{S I(n+1)}\right)$ given $S I(n)$. The actual (identified) $S I(n+1)$ value might be away from the middle of this distribution, and this difference over time going forward is the variability in which we are interested. Thus, variability was defined by the position of the realised eventual $S I(n+1)$ value relative to its predicted distribution $\hat{f}_{S I(n+1)}$. We will call $\hat{F}_{S I(n+1)}^{-1}(S I(n+1))$ (i.e. which percentile was the actual SI value on its predicted distribution) the "hit percentile". Optimally, it should be 50, high values indicate underestimated, low values indicate overestimated insulin sensitivity. The position of hit percentile was qualified numerically through two approaches. First, the hit percentile was compared to 50 (representing the median) with the squared difference characterizing the "error" in the prediction. This method was called the quadratic penalty. Secondly, deviations were penalized penalized asymmetrically by a one-sided threshold penalty that takes the value of 1 if the hit percentile is larger then 90, 0 otherwise. Hence, these metrices represent different physiological considerations in assessing variability, and the relationship between them was also investigated.

An overall variability score is obtained for a given patient by averaging these values across the time series of $S I$ values. For the one-sided threshold penalty, this averaging simply calculates the ratio of greater-then-90 percentile outcomes. This analysis was also carried out for daily windows during the stay in ICU.

An overall variability score can be calculated for a given diagnosis group by averaging the overall variability scores for only the patients belonging to that group. However, if the patients' length of stay differs, simple arithmetic averaging assigns unequal weights for each patient. Hence, only series of equal length were averaged, as described in the next paragraph.

Results are presented for the first 24 hours ("Day 1"), second 24 hours ("Day 2"), third 24 hours ("Day 3"), and remaining time in ICU ("Day 4 and onwards"). As a consequence, patients with less then 24 hours of data were excluded, leaving $n=261$ patients. These patients together had 47,836 hours and individual $S I$ values. Diagnosis groups were created based on the Apache III codes: Cardio (C), Gastro (G), All other (O), with Operative (Op) and Non-operative (NOp) breakdown in every category. This grouping is medically relevant and ensures proper sample sizes in every group to aid the robustness of the further statistical tests. Table 1 shows the distribution (according to length-of-stay and diagnosis group) and the most important demographic indicators of the patients.
Table 2. $p$-values of Kruskal-Wallis-test for the equality of average SI variability across diagnosis groups segregated according to day

\begin{tabular}{|c|c|c|}
\hline & One-sided threshold & Quadratic \\
\hline Day 1 & 0.1809 & 0.02234 \\
Day 2 & 0.1814 & 0.02094 \\
Day 3 & 0.9702 & 0.6884 \\
Day 4 and onwards & 0.1352 & 0.6499 \\
\hline
\end{tabular}

The distribution of the per-patient average penalty scores for a given diagnosis group and day was illustrated with boxplots (see Chambers et al. (1983) and Velleman et al. (1981)).

To investigate if the differences in $S I$ variability are significant, we used Kruskal-Wallis-tests To avoid potential difficulties with using repeated-measures ANOVA and the usage of the more complicated mixed-effects models, we split the data according to days, and performed separate analyses. Thus, the ANOVA-type test could be still applied.

To assess which diagnosis groups are differing, post-hoc testing was carried out using Tukey's Honestly Significant Differences (HSD) method, see Hsu (1996).

The preprocessing of the data was performed under Mathworks Matlab (version 2009a, see The MathWorks Inc. (2009)). Statistical analysis was performed under the $\mathrm{R}$ statistical program package (see R Development Core Team (2011)), version 2.14.2.

\section{RESULTS AND DISCUSSION}

Figure 1 shows the distribution of the per-patient overall variability metrics in different diagnosis groups, segregated according to ICU day and diagnosis group. Onesided threshold penalties exhibit much larger, typically positively-skewed variations. There is a slight trend in the central tendency, as median variability appears to decrease as time increases. A trend towards reduced spread in the variabilities over time is more pronounced.

In contrast, quadratic penalties are much more concentrated, and have a smaller coefficient of variation. The continuous lowering of variabilities in every group is also seen, but a reduction in spread is not as pronounced. The two metrics are consistent in assigning "higher" and "lower" variabilities similarly, albeit, on different scales.

Significance of the between-diagnosis group differences per-day according to both variability metrics is shown on Table 2 .

It can be seen there are no significant differences in SI variability according to diagnosis group on Day 3 and after, no matter which metric is used. There are no significant differences at all (on either day) according to the one-sided threshold penalty, however, there are significant differences on Day 1 and on Day 2 when the quadratic penalty is employed. (The former observation can be explained by the higher spread of per-patient variability metrics as seen on Figure 1.)

For the two cases, where significant difference was detected (Day 1 and Day 2 with quadratic penalty) post hoc testing 
Table 1. The distribution (according to length-of-stay and diagnosis group) and the most important demographic indicators of the patients. Data are shown in an $\mathrm{n}$, age, percentage of females format, with age statistics arranged in Mean (Median) \pm SD (IQR) manner. Columns indicate minimum (and not exact) length-of stay, so the same patient may appear in several cells.

\begin{tabular}{|c|c|c|}
\hline & Day 1 & Day 2 \\
\hline NonOP - Cardio & $28,59.5(61.5) \pm 16.5(24), 35.7$ & $18,58.4(59.5) \pm 16.1(19), 38.9$ \\
OP - Cardio & $35,72.9(73) \pm 7.12(10.8), 22.9$ & $21,72.9(73) \pm 6.54(10), 23.8$ \\
NonOP - Gastro & $16,64.3(67) \pm 12.8(15), 25$ & $13,64.4(71) \pm 14.2(18.5), 23.1$ \\
OP - Gastro & $42,67.9(72) \pm 12.4(13), 35.7$ & $29,69.9(72) \pm 10.8(11.3), 27.6$ \\
NonOP - All other & $119,54.7(59) \pm 18(27), 46.2$ & $101,54.5(59) \pm 18(28), 42.6$ \\
OP - All other & $21,50.8(56) \pm 19.2(31), 38.1$ & $16,54.9(57.5) \pm 18.5(31), 43.8$ \\
\hline & Day 3 & Day 4 and onwards \\
\hline NonOP - Cardio & $11,64.2(63) \pm 10.6(16.3), 18.2$ & $11,64.2(63) \pm 10.6(16.3), 18.2$ \\
OP - Cardio & $18,73.2(73.5) \pm 6.46(9), 27.8$ & $18,73.2(73.5) \pm 6.46(9), 27.8$ \\
NonOP - Gastro & $13,64.4(71) \pm 14.2(18.5), 23.1$ & $13,64.4(71) \pm 14.2(18.5), 23.1$ \\
OP - Gastro & $23,69.2(71) \pm 9.46(11.5), 26.1$ & $23,69.2(71) \pm 9.46(11.5), 26.1$ \\
NonOP - All other & $88,54.2(58) \pm 17.9(26.5), 45.5$ & $88,54.2(58) \pm 17.9(26.5), 45.5$ \\
OP - All other & $15,54.7(57) \pm 19.1(33.5), 40$ & $15,54.7(57) \pm 19.1(33.5), 40$ \\
\hline
\end{tabular}

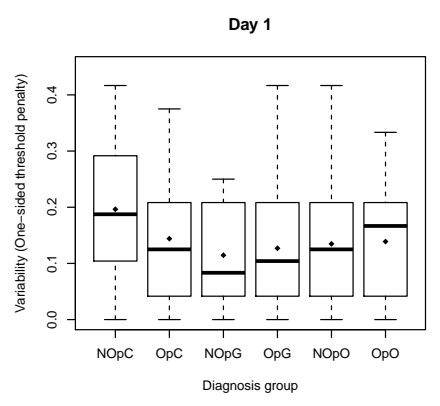

Day 1

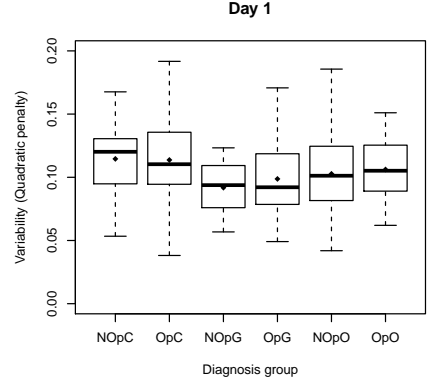

Day 2

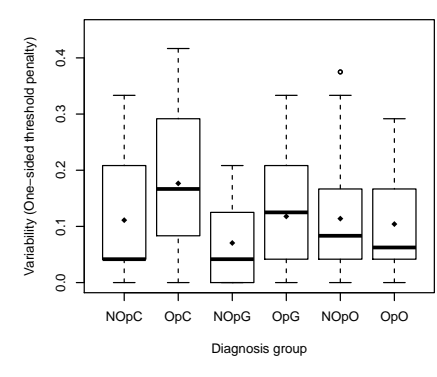

Day 2

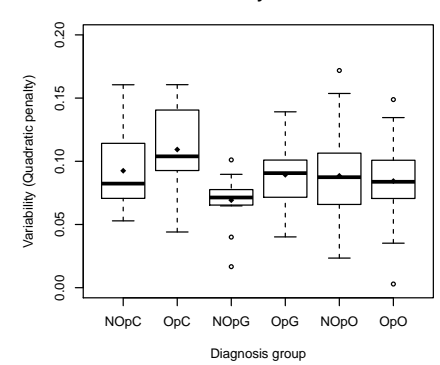

Day 3

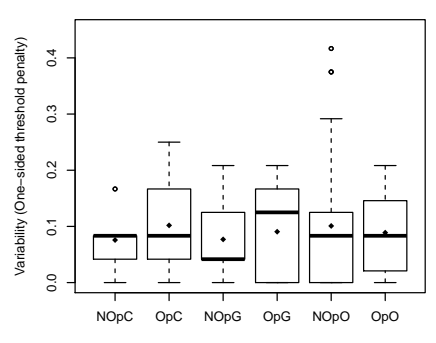

Day 3

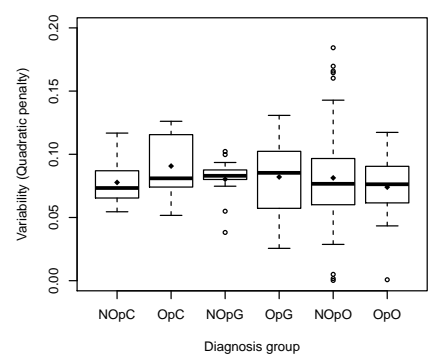

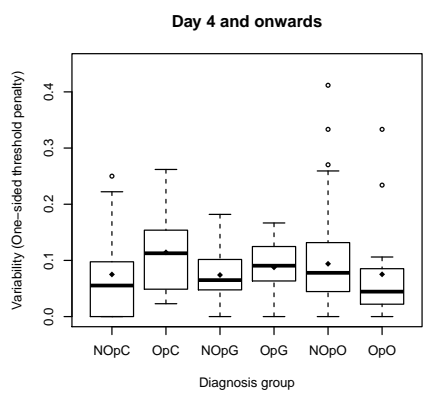

Day 4 and onwards

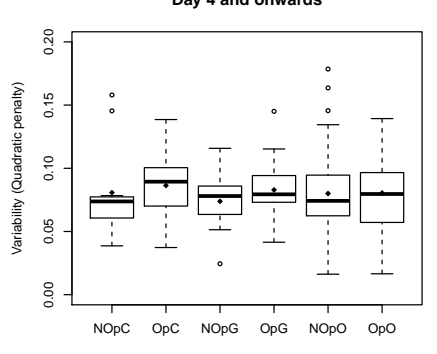

Diagnosis group

Fig. 1. Boxplots of per-patient overall variability scores segregated according to day and diagnosis group. Upper row shows one-sided threshold penalty metric, while lower row shows the quadratic penalty metric. Markers within boxes indicate mean, whiskers extend to the furthest observation no farther then 1.5IQR from the median.

was employed (Tukey's HSD for the classical ANOVA). Results are shown on Table 3.

For Day 1, no significant pairwise difference can be detected, on Day 2, Non-operative Gastro and Operative Cardio was significantly different $(p=0.00472)$, while Non-operative All other and Operative Cardio was very close to significance $(p=0.06305)$.

The falling trend of variability according to the one-sided threshold penalty indicates a decreasing chance of hypoglycemia (that can be attributed to large rises in insulin sensitivity over short time periods) as days of ICU stay increase. The overall risk of increased variability of both forms (one-sided and quadratic metrics) by diagnostic category is highest for Cardio patient groups matching the increased hypoglycemia observed in glycemic control studies in these cohorts (e.g. Preiser et al, 2010). The overall higher variability (according to quadratic measure) on Day 1 in all groups is also reflective of increased hypoglycemia and variability reported in most glycemic control studies Griesdale et al (2009).

As far as the physiological explanation of the phenomenon is concerned, it can be most likely linked to the counterregulatory and oxidative stress responses, and inflammatory acute immune response that is often observed in hyperglycemic critically ill patients. The fact that variability has a declining trend over days 1-4 - possibly as the acute phase passes - also matches expectations and physiological observations. 
Table 3. $p$-values for the post-hoc testing of the significant differences (Day 1 and Day 2 with quadratic penalty)

\begin{tabular}{|c|c|c|c|c|c|c|c|c|}
\hline \multirow{2}{*}{$\begin{array}{l}\text { Compared } \\
\text { pair }\end{array}$} & \multicolumn{4}{|c|}{ Day 1} & \multicolumn{4}{|c|}{ Day 2} \\
\hline & Estimate & Std. Error & $t$ value & $\operatorname{Pr}(>|t|)$ & Estimate & Std. Error & $t$ value & $\operatorname{Pr}(>|t|)$ \\
\hline $\mathrm{OpC}-\mathrm{NOpC}==0$ & -0.000871 & 0.007403 & -0.118 & 1.000 & 0.0167845 & 0.0100620 & 1.668 & 0.53874 \\
\hline $\mathrm{NOpG}-\mathrm{NOpC}==0$ & -0.022773 & 0.009151 & -2.489 & 0.124 & -0.0232665 & 0.0114018 & -2.041 & 0.30979 \\
\hline $\mathrm{OpG}-\mathrm{NOpC}==0$ & -0.015855 & 0.007124 & -2.226 & 0.219 & -0.0031855 & 0.0093997 & -0.339 & 0.99934 \\
\hline $\mathrm{NOpO}-\mathrm{NOpC}==0$ & -0.011849 & 0.006133 & -1.932 & 0.371 & -0.0040197 & 0.0080145 & -0.502 & 0.99571 \\
\hline $\mathrm{OpO}-\mathrm{NOpC}==0$ & -0.008392 & 0.008429 & -0.996 & 0.914 & -0.0081472 & 0.0107632 & -0.757 & 0.97213 \\
\hline $\mathrm{NOpG}-\mathrm{OpC}==0$ & -0.021902 & 0.008812 & -2.486 & 0.125 & -0.0400510 & 0.0110550 & -3.623 & 0.00472 \\
\hline $\mathrm{OpG}-\mathrm{OpC}==0$ & -0.014984 & 0.006683 & -2.242 & 0.212 & -0.0199700 & 0.0089758 & -2.225 & 0.22025 \\
\hline $\mathrm{NOpO}-\mathrm{OpC}==0$ & -0.010978 & 0.005615 & -1.955 & 0.357 & -0.0208042 & 0.0075129 & -2.769 & 0.06305 \\
\hline $\mathrm{OpO}-\mathrm{OpC}==0$ & -0.007521 & 0.008060 & -0.933 & 0.933 & -0.0249317 & 0.0103951 & -2.398 & 0.15341 \\
\hline OpG $-\mathrm{NOpG}==0$ & 0.006918 & 0.008578 & 0.806 & 0.964 & 0.0200809 & 0.0104557 & 1.921 & 0.37789 \\
\hline $\mathrm{NOpO}-\mathrm{NOpG}==0$ & 0.010924 & 0.007775 & 1.405 & 0.711 & 0.0192468 & 0.0092304 & 2.085 & 0.28641 \\
\hline $\mathrm{OpO}-\mathrm{NOpG}==0$ & 0.014381 & 0.009689 & 1.484 & 0.661 & 0.0151193 & 0.0116968 & 1.293 & 0.77735 \\
\hline $\mathrm{NOpO}-\mathrm{OpG}==0$ & 0.004006 & 0.005241 & 0.764 & 0.971 & -0.0008342 & 0.0065995 & -0.126 & 0.99999 \\
\hline $\mathrm{OpO}-\mathrm{OpG}==0$ & 0.007463 & 0.007804 & 0.956 & 0.927 & -0.0049617 & 0.0097554 & -0.509 & 0.99542 \\
\hline $\mathrm{OpO}-\mathrm{NOpO}==0$ & 0.003457 & 0.006911 & 0.500 & 0.996 & -0.0041275 & 0.0084289 & -0.490 & 0.99617 \\
\hline
\end{tabular}

\section{CONCLUSION}

Variability in insulin sensitivity peaks on Day 1 across diagnostic groups, as well as metrics, but differences between diagnostic groups diminish as time passes.

Quadratic penalty exhibits smaller differences between diagnostic groups, but also smaller variations within groups. Thus, it can be concluded that overall variability is more stable across patients, in contrast to the risk for unexpected rises in $S I$ which shows much higher inter-patient variability.

As a result of this, there are no overall significant differences between diagnostic groups when one-sided threshold penalty is considered, i.e. diagnosis groups are equivalent from the risk point of view (when only unexpected rises in $S I$ are considered).

There are, however, significant differences when quadratic penalty (i.e. overall variability) is considered; it is suggested the operative cardiac patients exhibit higher variability. This is in concordance with the literature findings and the possible pathophysiological explanations.

\section{REFERENCES}

J. S. Krinsley. Association between hyperglycemia and increased hospital mortality in a heterogeneous population of critically ill patients. Mayo Clin Proc, 78:1471-1478, 2003.

K. C. McCowen, A. Malhotra, B. R. Bistrian. Stressinduced hyperglycemia. Crit Care Clin, 17:107-124, 2001.

D. E. Griesdale, R. J. de Souza, R. M. van Dam, D. K. Heyland, D. J. Cook, A. Malhotra, R. Dhaliwal, W. R. Henderson, D. R. Chittock, S. Finfer, D. Talmor. Intensive insulin therapy and mortality among critically ill patients: a meta-analysis including NICE-SUGAR study data. CMAJ, 180:821-827, 2009.

S. Bagshaw, R. Bellomo, M. Jacka, M. Egi, G. Hart, C. George, t. A. C. M. Committee. The impact of early hypoglycemia and blood glucose variability on outcome in critical illness. Critical Care, 13:R91, 2009.

M. Egi, R. Bellomo, E. Stachowski, C. J. French, G. Hart. Variability of blood glucose concentration and short- term mortality in critically ill patients. Anesthesiology, 105:244-252, 2006.

J. S. Krinsley. Glycemic variability: a strong independent predictor of mortality in critically ill patients. Crit Care Med, 36:3008-3013, 2008.

J. S. Krinsley. Effect of an intensive glucose management protocol on the mortality of critically ill adult patients. Mayo Clin Proc, 79:992-1000, 2004.

J. G. Chase, G. Shaw, A. Le Compte, T. Lonergan, M. Willacy, X.-W. Wong, J. Lin, T. Lotz, D. Lee, C. Hann. Implementation and evaluation of the SPRINT protocol for tight glycaemic control in critically ill patients: a clinical practice change. Critical Care, 12:R45, 2008.

G. Van den Berghe, P. Wouters, F. Weekers, C. Verwaest, F. Bruyninckx, M. Schetz, D. Vlasselaers, P. Ferdinande, P. Lauwers, and R. Bouillon. Intensive insulin therapy in the critically ill patients. N Engl J Med, 345:1359-1367, 2001.

M. P. Casaer, D. Mesotten, G. Hermans, P. J. Wouters, M. Schetz, G. Meyfroidt, S. Van Cromphaut, C. Ingels, P. Meersseman, J. Muller, D. Vlasselaers, Y. Debaveye, L. Desmet, J. Dubois, A. Van Assche, S. Vanderheyden, A. Wilmer, G. Van den Berghe. Early versus late parenteral nutrition in critically ill adults. N Engl J Med, 365:506$517,2011$.

F. M. Brunkhorst, C. Engel, F. Bloos, A. Meier-Hellmann, M. Ragaller, N. Weiler, O. Moerer, M. Gruendling, M. Oppert, S. Grond, D. Olthoff, U. Jaschinski, S. John, R. Rossaint, T. Welte, M. Schaefer, P. Kern, E. Kuhnt, M. Kiehntopf, C. Hartog, C. Natanson, M. Loeffler, K. Reinhart. Intensive insulin therapy and pentastarch resuscitation in severe sepsis. N Engl J Med, 358:125139, 2008.

S. Finfer, D. R. Chittock, S. Y. Su, D. Blair, D. Foster, V. Dhingra, R. Bellomo, D. Cook, P. Dodek, W. R. Henderson, P. C. Hebert, S. Heritier, D. K. Heyland, C. McArthur, E. McDonald, I. Mitchell, J. A. Myburgh, R. Norton, J. Potter, B. G. Robinson, J. J. Ronco. Intensive versus conventional glucose control in critically ill patients. N Engl J Med, 360:1283-1297, 2009.

I. Mackenzie, S. Ingle, S. Zaidi, S. Buczaski. Tight glycaemic control: a survey of intensive care practice in large English hospitals. Intensive Care Med, 31:1136, 2005. 
D. Aragon. Evaluation of nursing work effort and perceptions about blood glucose testing in tight glycemic control. Am J Crit Care, 15:370-377, 2006.

J. G. Chase, S. Andreassen, K. Jensen, G. M. Shaw. Impact of Human Factors on Clinical Protocol Performance: A Proposed Assessment Framework and Case Examples. Journal of Diabetes Science and Technology, 2:409-416, 2008.

J. G. Chase, A. J. Le Compte, F. Suhaimi, G. M. Shaw, A. Lynn, J. Lin, C. G. Pretty, N. Razak, J. D. Parente, C. E. Hann, J.-C. Preiser, T. Desaive. Tight glycemic control in critical care - The leading role of insulin sensitivity and patient variability: A review and modelbased analysis. Computer Methods and Programs in Biomedicine, 102:156-171, 2011.

E. S. Moghissi, M. T. Korytkowski, M. DiNardo, D. Einhorn, R. Hellman, I. B. Hirsch, S. E. Inzucchi, F. IsmailBeigi, M. S. Kirkman, G. E. Umpierrez. American Association of Clinical Endocrinologists and American Diabetes Association consensus statement on inpatient glycemic control. Diabetes Care, 32:1119-1131, 2009.

F. Weekers, A. P. Giulietti, M. Michalaki, W. Coopmans, E. Van Herck, C. Mathieu, G. Van den Berghe. Metabolic, endocrine, and immune effects of stress hyperglycemia in a rabbit model of prolonged critical illness. Endocrinology, 144:5329-5338, 2003.

J. Lin, D. Lee, J. G. Chase, G. M. Shaw, A. Le Compte, T. Lotz, J. Wong, T. Lonergan, C. E. Hann. Stochastic modelling of insulin sensitivity and adaptive glycemic control for critical care. Computer Methods and Programs in Biomedicine, 89:141-152, 2008.

J. M. Chambers, W. S. Cleveland, B. Kleiner, P. A. Tukey. Graphical Methods for Data Analysis. Wadsworth \& Brooks/Cole, 1983.

P. F. Velleman, D. C. Hoaglin. Applications, Basics, and Computing of Exploratory Data Analysis. Duxbury Press, Pacific Grove, 1981.

J. Hsu. Multiple Comparisons: Theory and Methods. Chapman and Hall/CRC, Boca Raton, 1996.

The MathWorks Inc. MATLAB version 2009b. The MathWorks Inc., Natick, Massachusets, 2009.

$\mathrm{R}$ Development Core Team. R: A Language and Environment for Statistical Computing. R Foundation for Statistical Computing, Vienna, Austria, 2011. 